\title{
Modified shell technique for vertical ridge augmentation - Case report
}

\section{Solanki A ${ }^{1}$, Phadnaik $M^{2}$, Shirude $T^{3}$, Naik $D^{4}$}

\section{Affiliations:}

1. Holistic dental care, JP Road, Andheri, Mumbai Private practitioner and Consultant Periodontist.

2. Dean Government Dental College and Hospital, Nagpur, India.

3. Suhasya dental care, Nashik, Private practitioner and consultant periodontist 4. Dental Point, Kurla, Mumbai. Private practitioner and consultant Prosthodontist.

Corresponding author:

Dr. Ankit Solanki

Holistic dental care, Chandrakamal Bld,

mukund Patil lane, Andheri west, Mumbai solankiankit16@gmail.com

\section{ABSTRACT}

Purpose: The placement of an implant is hindered by the absence of an adequate volume of bone. Vertical ridge augmentation is one of the most difficult and unpredictable procedures. The purpose of this report is to present a clinical case of vertical ridge reconstruction around a single tooth with a slight variation in the described 'modified shell technique'.

Case report: the patient had presented with $8.5 \mathrm{~mm}$ vertical ridge deficiency in the region of central incisor \#21. An autogenous graft was harvested from the symphysis. The cortical plate was thinned into the shell and divided into two parts to be placed on both buccal and lingual aspects. They were secured at the desired location using titanium screw as the traction screw since there was a lack of availability of the native bone. Remarkable vertical augmentation of the alveolar bone with minimal bone resorption was noticed on re-entry at 6 months.

Conclusions: This technique shows an alternate method of vertical augmentation over a small edentulous span presenting minimal amount of bone remaining to secure the grafting material.

\section{KEYWORDS}

Vertical ridge augmentation, anterior reconstruction, autologous-graft, modified shell technique 


\section{INTRODUCTION}

Alveolar ridge resorption, regardless of the cause, is an inevitable sequel of tooth loss. ${ }^{1}$ The rate and magnitude of the resorption, however, may vary depending upon the site, the etiology, the soft and hard tissue quality, intensity of force it is exposed to etc. The anterior aesthetic region, which often presents with a thin buccal cortical plate, has been shown to exhibit the greatest amount of resorption, especially when compounded with a thin periodontal biotype. ${ }^{2}$ The maxillary anterior region is also the most susceptible to trauma, which may lead, not only to tooth loss, but the fracture of the buccal cortical plate, resulting in a vertically and horizontally deficient edentulous span. Implant supported prosthetic rehabilitation in such cases requires correction of the ridge defect, in both the horizontal and vertical directions, to enable 3D positioning of the implants for optimum aesthetics and function. ${ }^{3}$

Various methods have been suggested for alveolar ridge augmentation like Ridge split technique ${ }^{4}$, Guided bone regeneration $(\mathrm{GBR})^{5}$, Block graft $^{6}$, Sandwich technique ${ }^{7}$, Distraction osteogenesis ${ }^{8}$ and various modifications of these techniques.

Horizontal ridge augmentation has been demonstrated to be more predictable due to the presence of either or both the palatal/lingual and buccal cortical plates, which help stabilize the graft as well as provide a source for neoangiogenesis. ${ }^{10,11}$ In comparison, vertical ridge augmentation is less predictable.12, ${ }^{13}$ Use of autogenous bone is considered the gold standard for grafting and thus is the most preferred graft material in cases of vertical ridge augmentation. M Stimmelmayr et al. published a report on horizontal ridge augmentation using shells of cortical plate and found minimal ridge resorption with this technique. ${ }^{14}$ In the present case, a similar method was used and modified for achieving vertical augmentation of the ridge.

\section{CASE REPORT}

A 26-year-old male patient reported to the Department of Periodontology with a missing maxillary left central incisor. The patient gave a history of a traumatic injury leading to avulsion of the referred tooth 4-5 years back, which was treated by reimplantation of the tooth in its socket. The tooth, reportedly, was stable for about 4 years, but then showed progressive mobility over a period of time. It was extracted 7 months prior to the patient's current visit for its replacement.

On intra-oral examination, the edentulous ridge was adjudged to be deficient in the region of central incisor \#21 (Figure1). A cone beam computed tomographic (CBCT) image showed a significant vertical defect in the region of central incisor \#21 extending labio-palatally (Figure 2). The Nasopalatine canal was present such that it covered a large part of the region of left central incisor. After radiographic evaluation, the height of the remaining alveolar ridge was $6.45 \mathrm{~mm}$ in the centre of the defect area, bucco-lingual width was $4.08 \mathrm{~mm}$ at the mid-crestal area and mesio-distal length was $8.49 \mathrm{~mm}$ at the most coronal aspect of the defect, $2 \mathrm{~mm}$ apical to cement-enamel junction of the adjacent teeth (Figure 3). However, the bone was present up to the coronal third of the teeth adjacent to the defect. This gave an inverse "V" shaped morphology to the defect.

Under local anaesthesia, a full thickness muco-periosteal flap

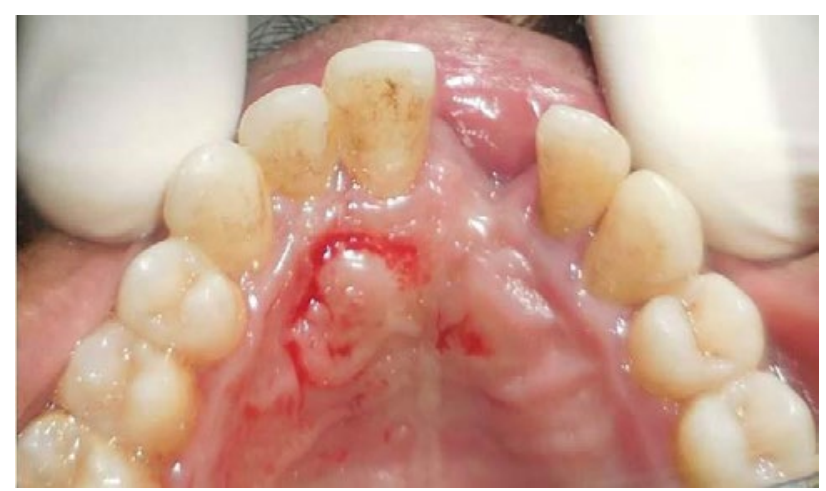

Figures 1. Intraoral photographs with palatal view of region of central incisor (\#21) having soft tissue deficiency.

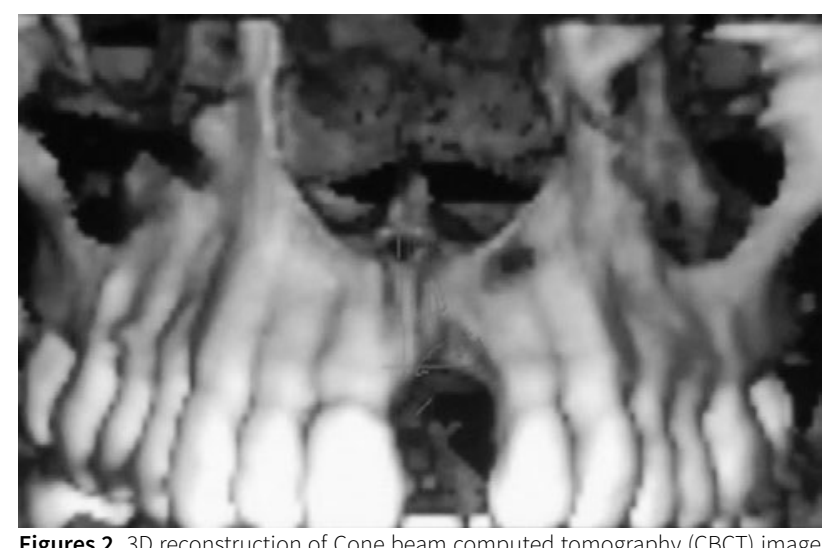

Figures 2. 3D reconstruction of Cone beam computed tomography (CBCT) image of central incisor (\#21) region having severe vertical ridge deficiency.
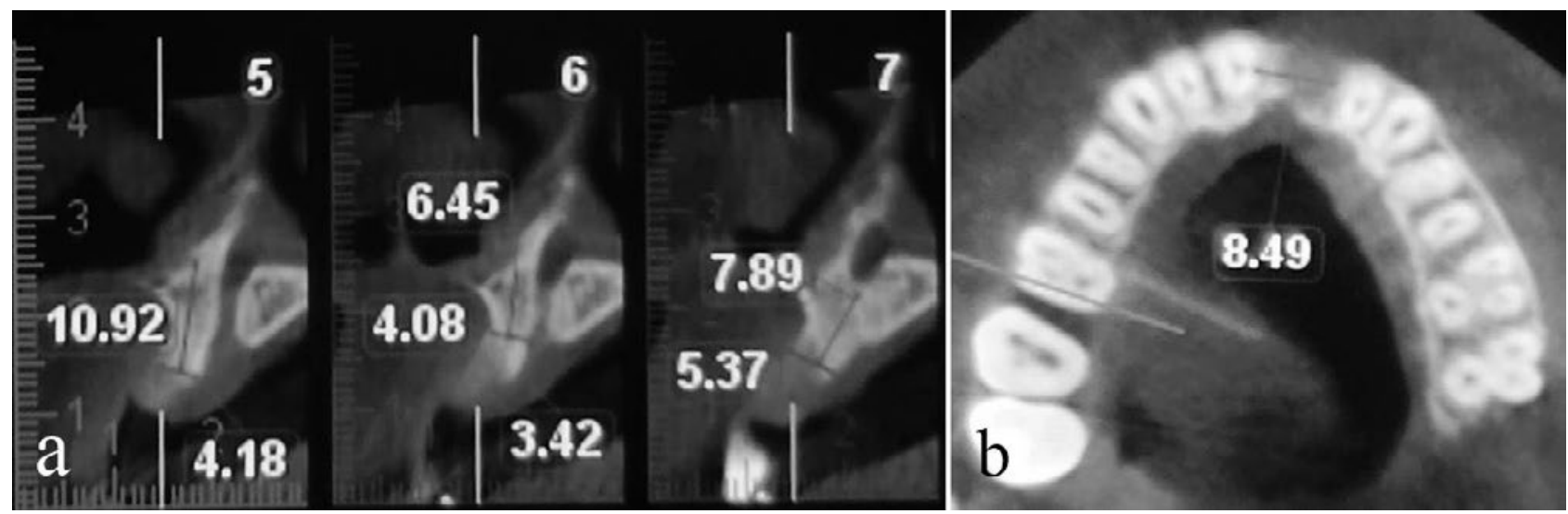

Figures 3. Cone beam computed tomography (CBCT) cross-sectional images showing central portion of the ridge of \#21 region having inadequate width and height for implant placement. 
was raised. A crestal incision was slightly palatally placed and two vertical incisions were placed, one distal to the maxillary right central incisor and the other, distal to the left lateral incisor not including the distal papillae. The inverse "V" shaped defect could then be visualised measuring about $10 \mathrm{~mm}$ from the most coronal portion of alveolar ridge to the deepest portion of the defect at centre of the ridge (Figures 4,5) The large incisive foramen was present on the mesiopalatal aspect. The nasopalatine nerve, however, was not curetted during the grafting procedure as the patient had refused consent for the same. A periosteal release incision was given to enable advancement of the buccal flap over the grafted site. The site was prepared for receiving the graft material by intra marrow penetration.

A block graft, approximately the apico-incisal and twice the

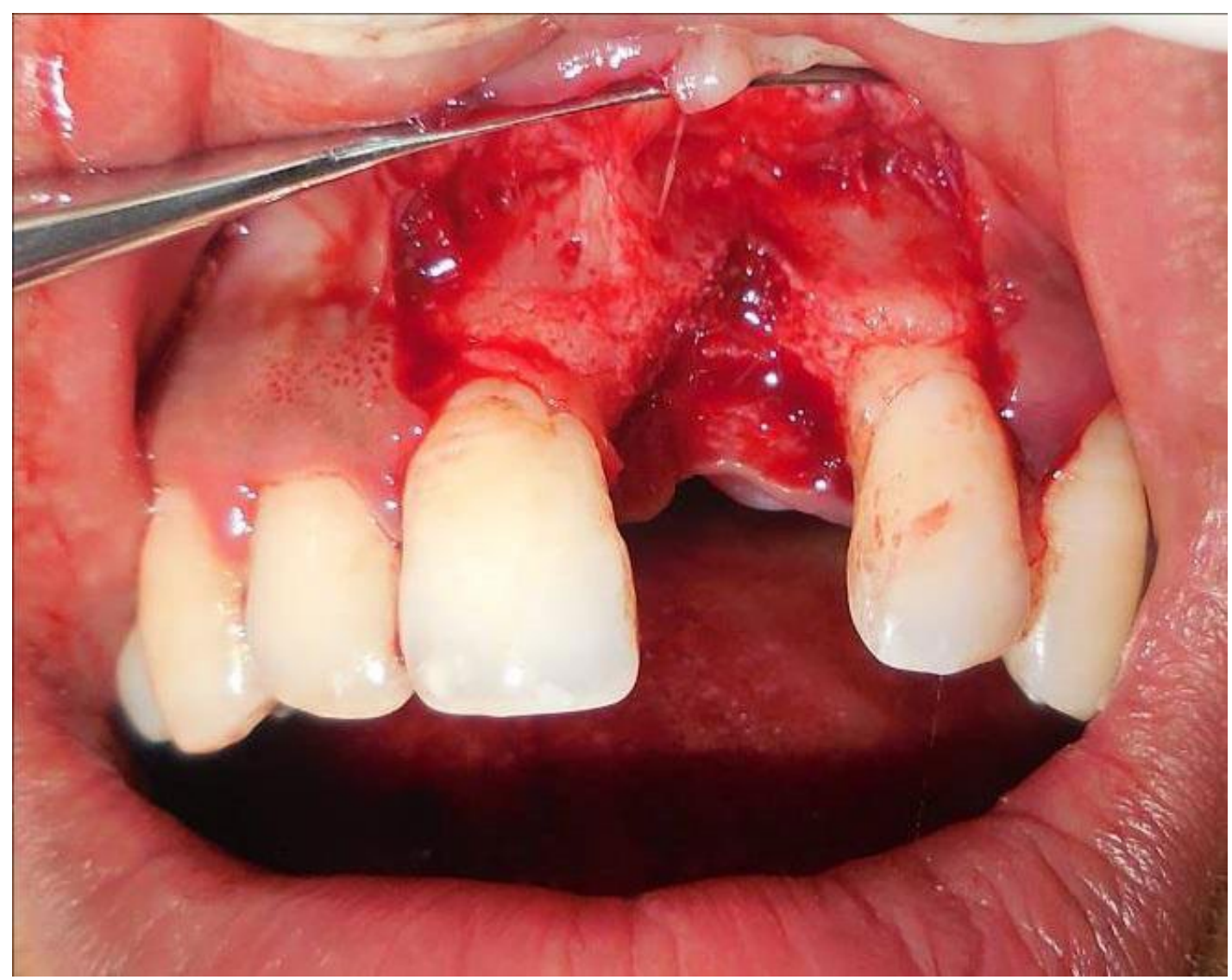

Figures 4. Alveolar ridge exposed. Inverted "V "shaped defect in \#21 region passing through buccal palatal.

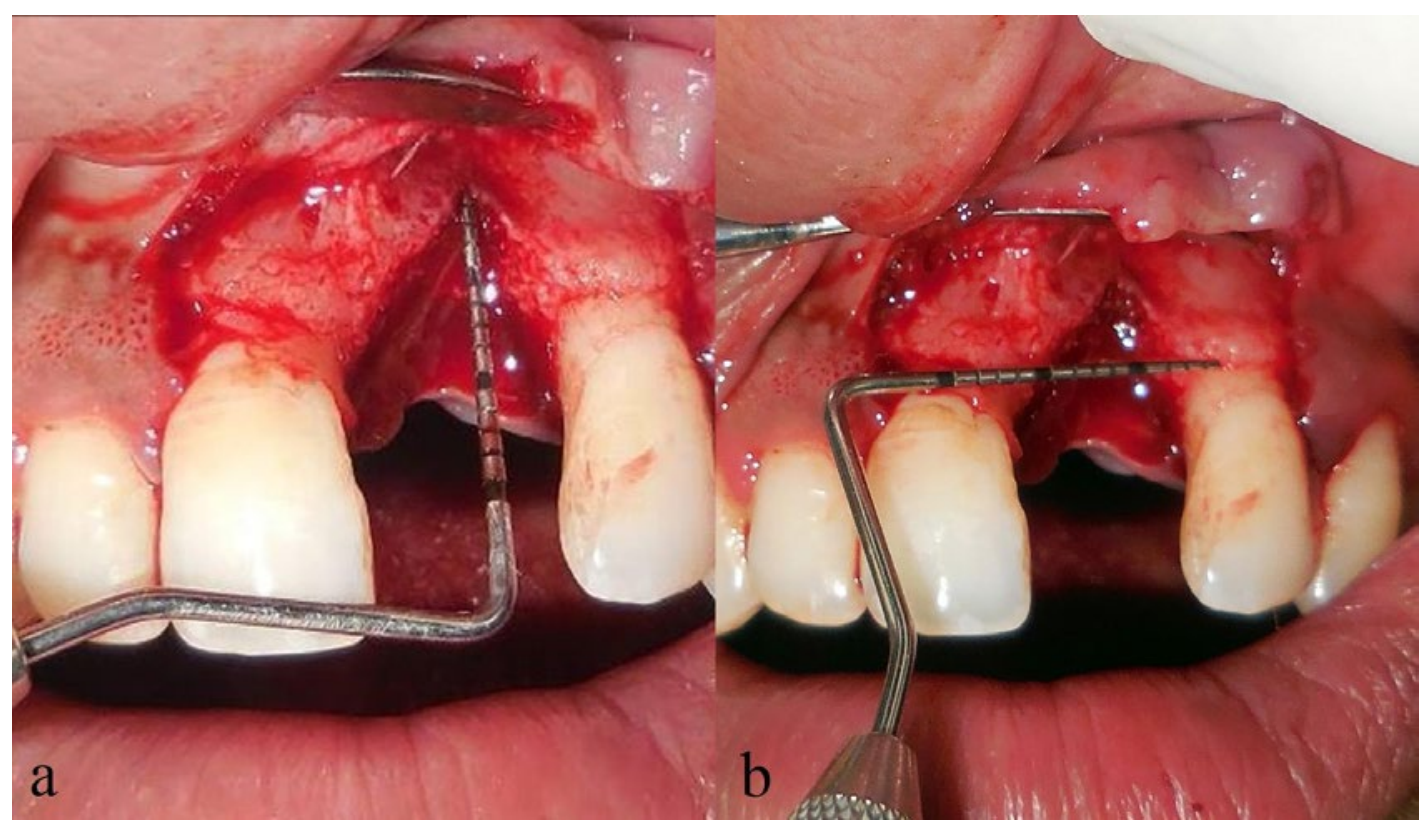

Figures 5. Vertical defect in \#21 region measuring about $10 \mathrm{~mm}$ in height and width at the centre of the ridge. 
mesio-distal dimension of the defect, was harvested from the symphysis region using a piezo-surgical unit (Acteon, Satelec, USA) (Figure 6). The cancellous bone was scraped off to obtain a shell of cortical bone that was further divided into two parts using a piezo-surgical insert for placement on either side of the defect (Figure 7). The donor site was packed with absorbable gelatine sponge.
The two cortical shells were trimmed to approximate the defect margins, the edges smoothened and adapted to the recipient site at the desired position. A central hole was drilled through the cortical plates to receive a titanium screw which anchored and secured the shells on either side of the defect snugly, without encroaching on the nasopalatine space (Figure 8). A mixture of freeze dried allograft (Tissue Bank, Tata Memorial Hospital, India)

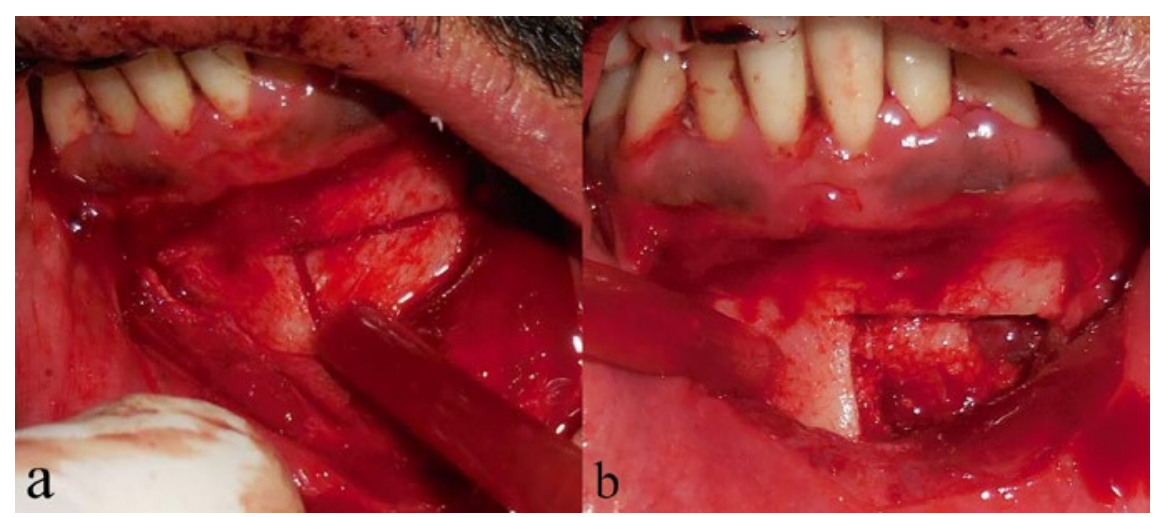

Figures 6. Exposed symphyseal area (donor site) for block graft harvesting with piezo-surgery device.

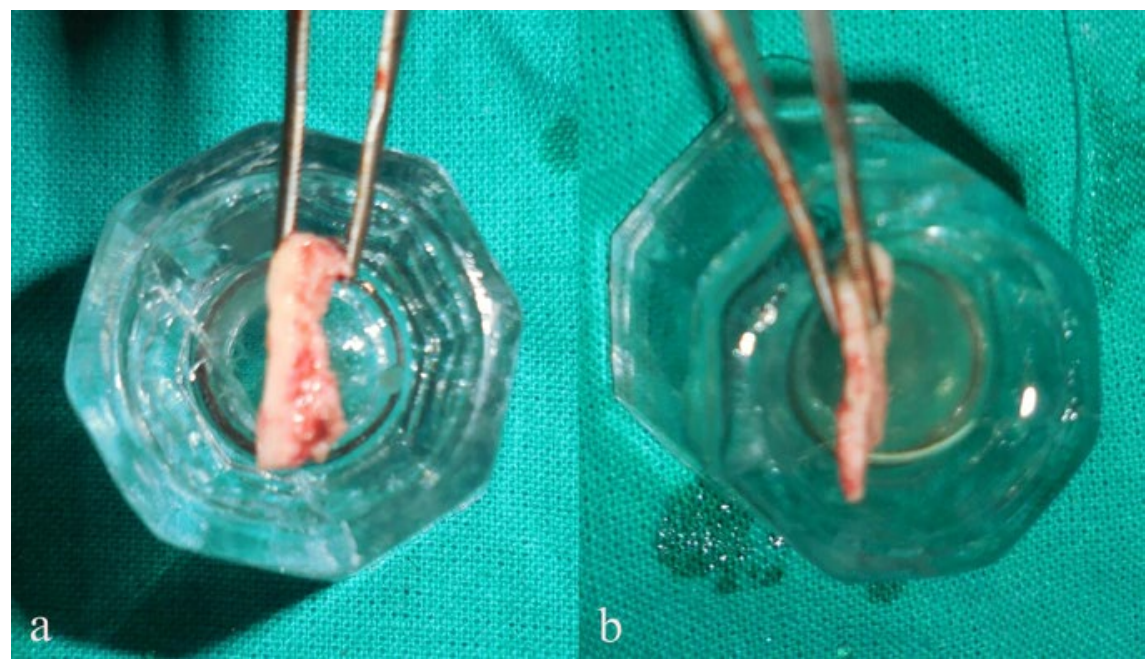

Figures 7. Block graft retrieved from symphyseal area (donor site). The cortical shell after separating cancellous part.

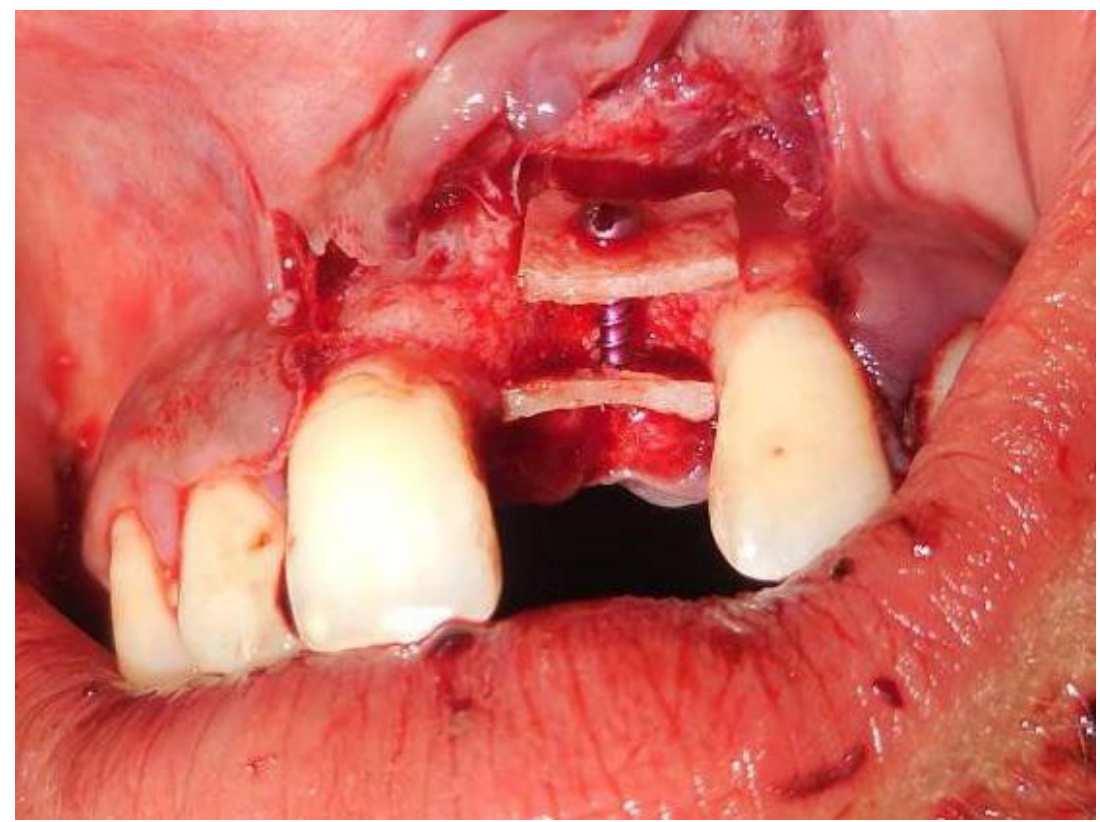

Figures 8. Cortical shells adapted to the recipient site with titanium screw. 
and cancellous bone obtained during the graft harvest previously was used to fill the space between the secured cortical shells and the grafted area was covered with a chorion membrane (Tissue Bank, Tata Memorial Hospital, India) (Figure 9). Both the donor and the recipient site were sutured with 3-0 silk. (Fig. 10) The post-operative drug regime included a combination of antibiotics and analgesics (Amoxicillin+ clavulanic acid $625 \mathrm{mg}$ twice daily for seven days and aceclofenac $100 \mathrm{mg}+$ paracetamol $500 \mathrm{mg}$ for 7 days. A $0.2 \%$ ) chlorhexidine mouthrinse for two weeks and strict postoperative instructions were given, and the patient was recalled for suture removal.

The sutures placed at the donor site were removed at 7 days and at the recipient site after 14 days. The healing at the recipient site was uneventful while there was suppuration at the donor site during suture removal. This was managed by irrigation with warm saline and povidone iodine. Antibiotics were continued for further 5 days. The site healed within a week with no further complication (Figure 11). The patient chose not to monitor the healing period. He was contacted monthly where there were no reports of complications during the entire 6-month period.

A 6-month post-operative CBCT showed a well healed site with adequate augmentation achieved in both the vertical and horizontal dimensions. After radiographic evaluation, the width of the alveolar ridge $2 \mathrm{~mm}$ below the crests was $6.79 \mathrm{~mm}$ and the
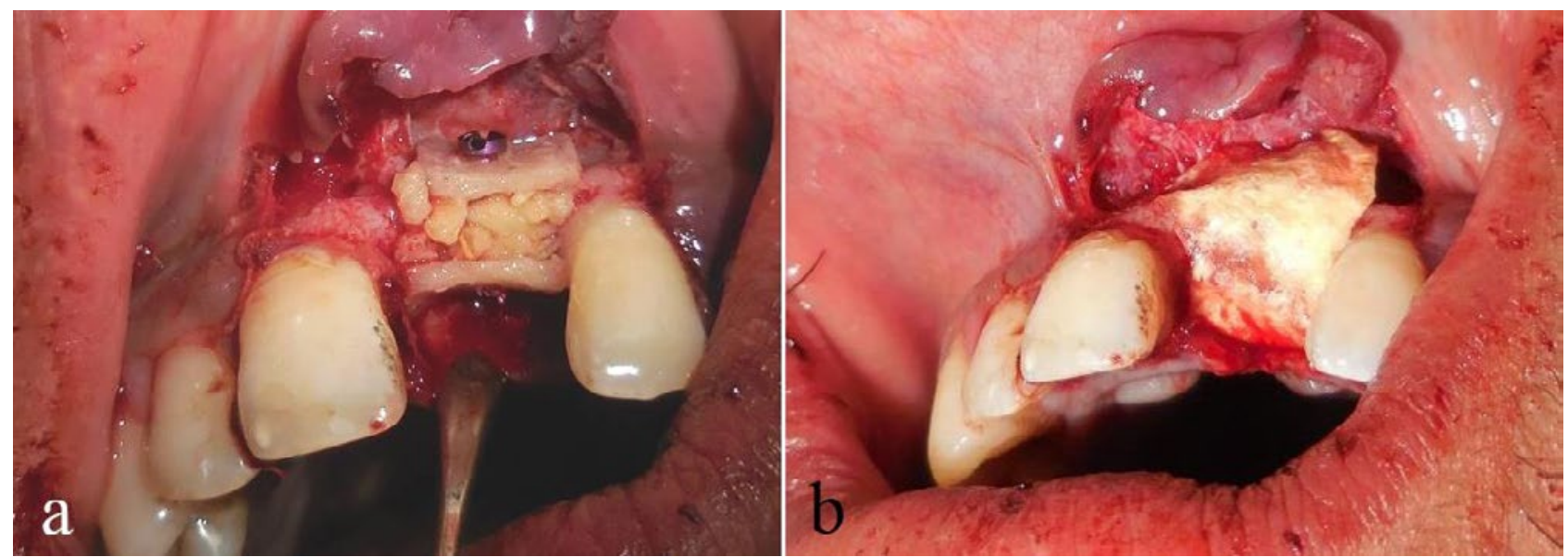

Figures 9. Grafted site filled with mixture of cancellous graft and FDBA covered with chorion membrane.
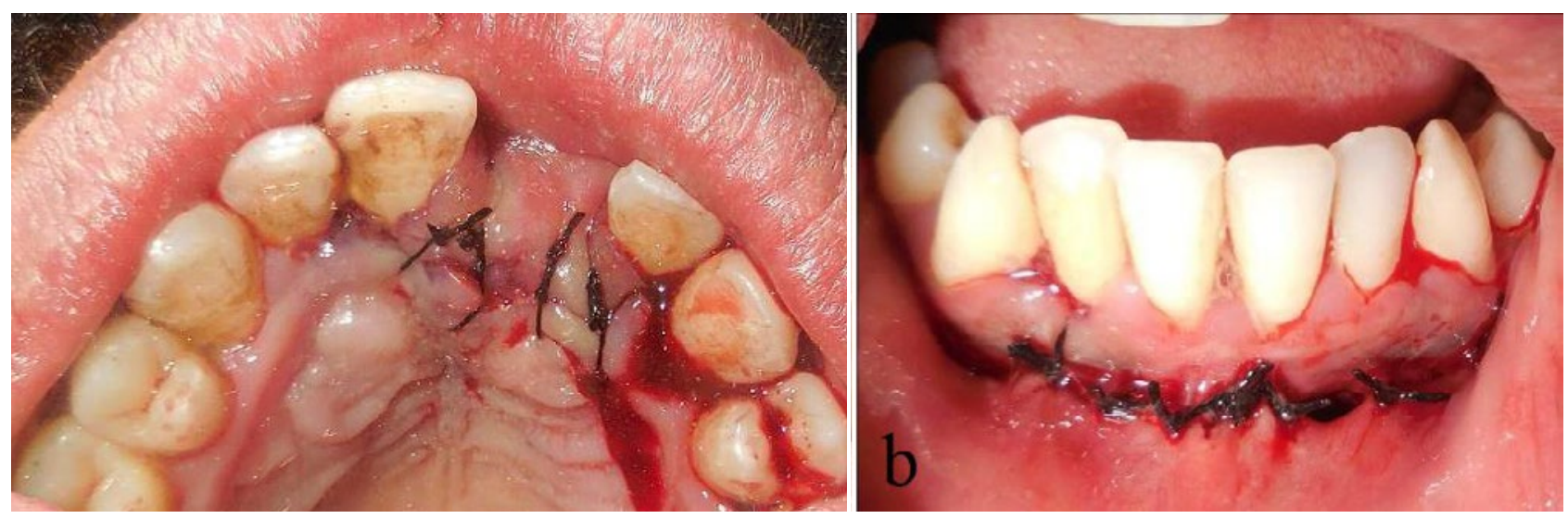

Figures 10. Suturing at the recipient and the donor site.
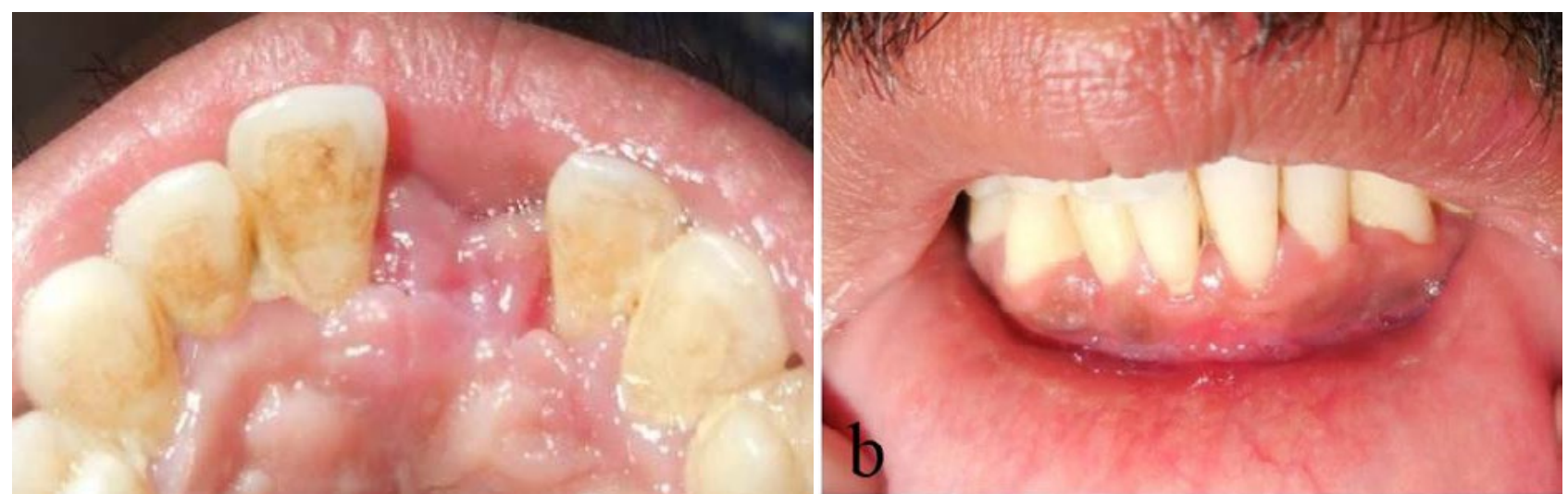

Figures 11. 14 days post-operative healing at the recipient and the donor site. 
height was $13.02 \mathrm{~mm}$. A full thickness flap was reflected with a crestal incision and the titanium screw was removed. Clinically, well healed, healthy bone with optimal incorporation of the graft and adequate augmentation of the soft as well as hard tissue was observed (Figure 12). A $3.5 \times 11.5$ implant (Osstem TSIII implant) was placed, and site was closed and allowed to heal for further 6 months (Figure 13). Implant was placed slightly buccally than ideal due to the presence of a large nasopalatine canal in the region. The presence of adequate bone buccally facilitated implant placement. There was presence of adequate soft tissue cover with a profile matching adjacent tooth, hence no soft tissue augmentation was performed.
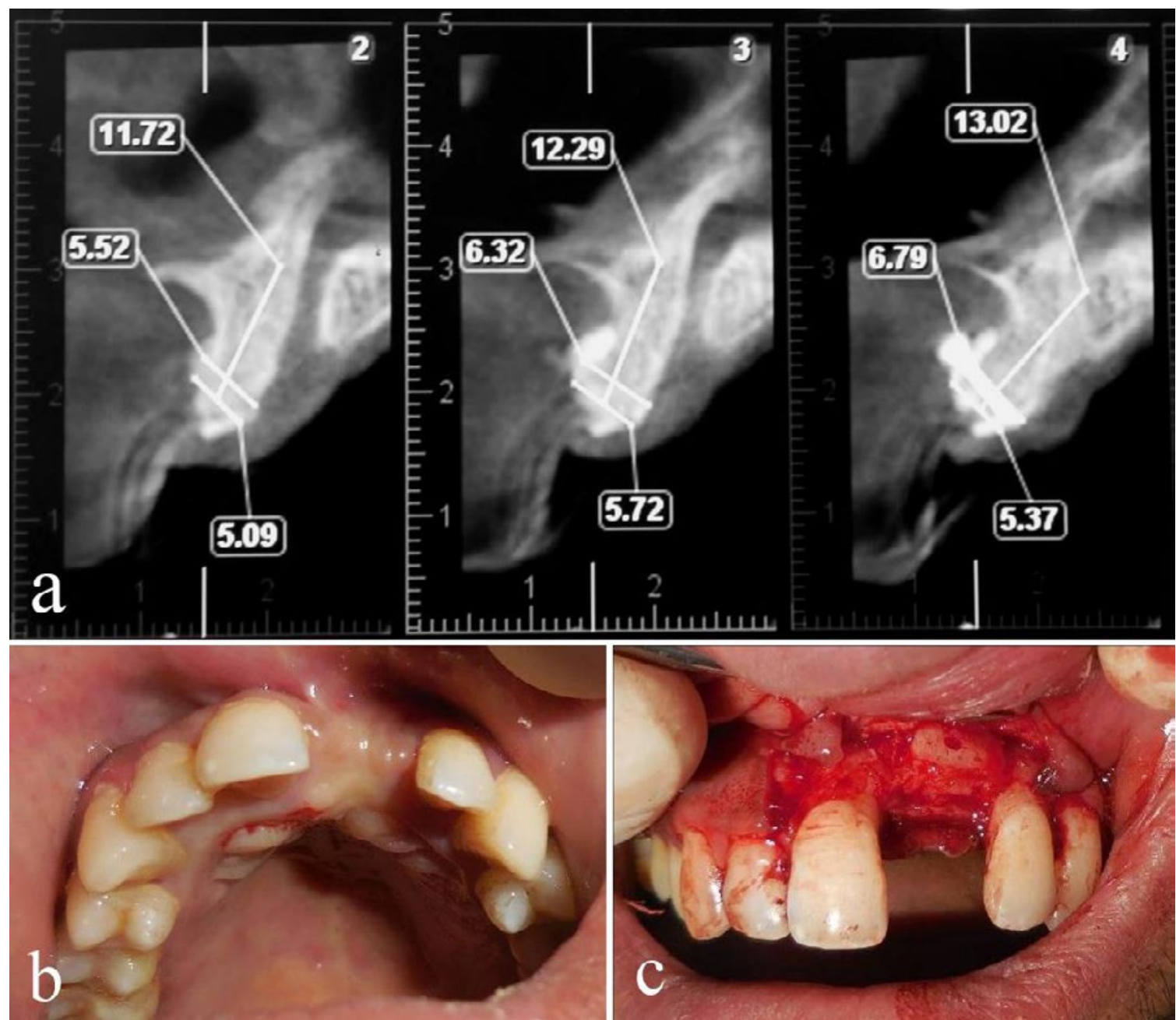

Figure 12. 6 months Post-operative with adequate soft and hard tissue augmentation of \#21 region.
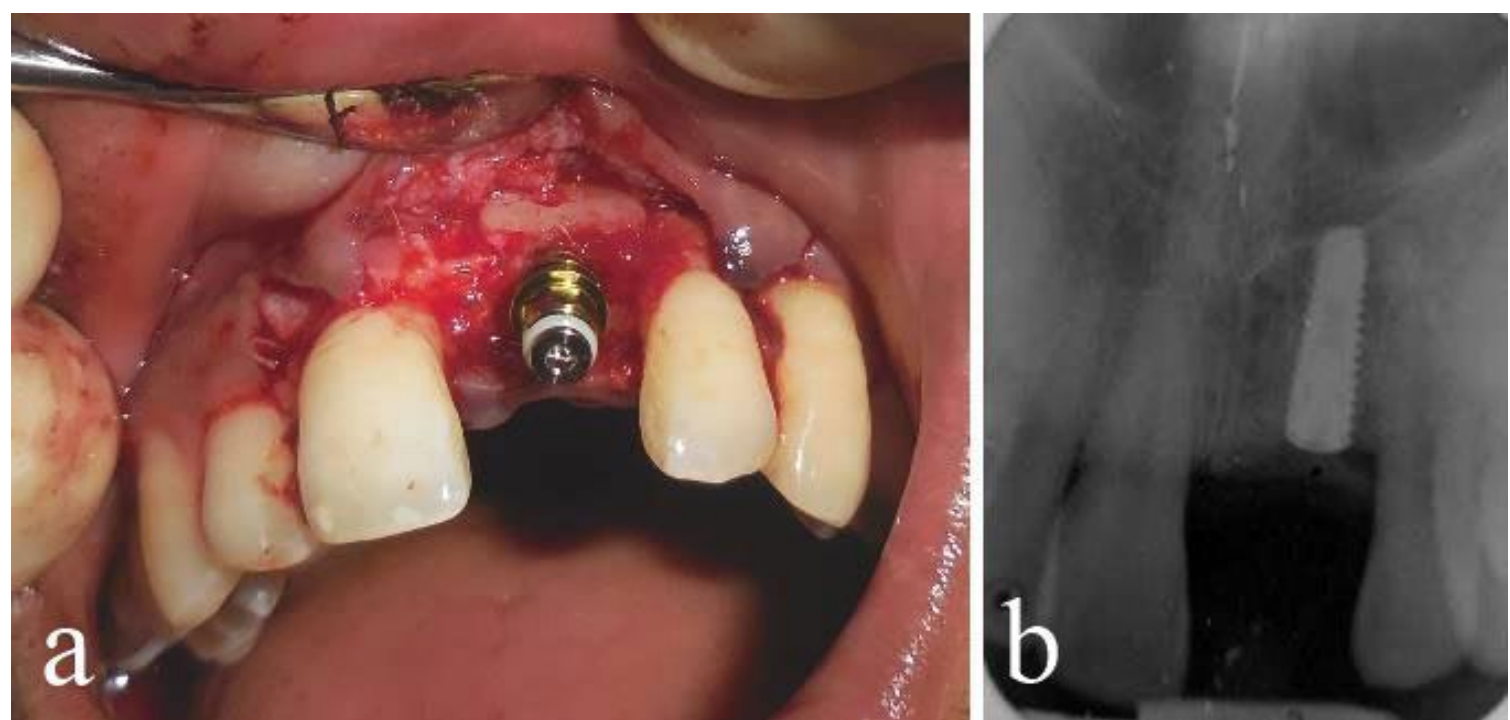

Figure 13. Placement of $3.5 \times 11.5 \mathrm{~mm}$ implant in \#21 region. 

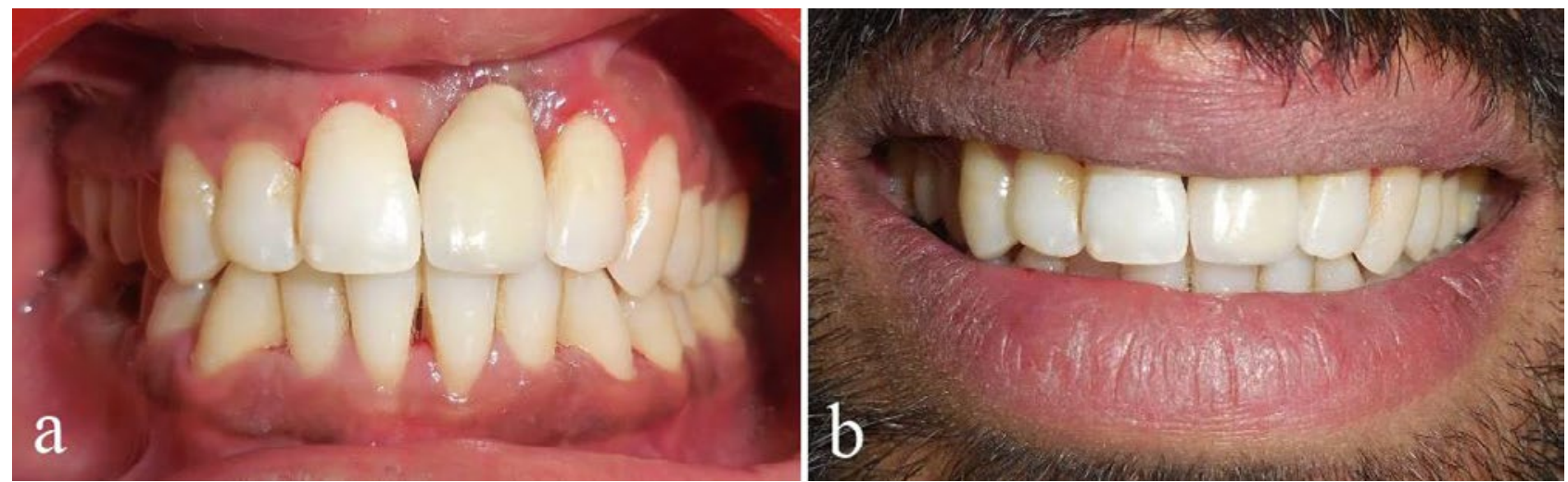

Figure 14. Implant supported cement retained metal-ceramic crown \#21

The definitive porcelain fused to metal crown restoration was delivered to the patient, 6 months after implant placement. There was a discrepancy in the gingival zenith compared to the contralateral tooth. But since the patient had a low lip-line this was not an aesthetic concern for the patient and the patient was satisfied with the prosthesis (Figure 14).

\section{DISCUSSION}

Three dimensional defects, such as in the present case, have traditionally been managed with block grafts since they provide stability. However, a critical concern in the use of block grafts has always been the ability of the graft to adapt to complex defect configuration and its vascularisation at the recipient site. Particulate grafts, on the other hand, are easier to adapt and are easily vascularised. The technique used in the current case employs a combination of block graft and guided bone regeneration such that, the cortical shells offer the stability of block grafts while being more resistant to resorption and consequent collapse, while the particulate graft allows for better revascularization. ${ }^{15}$ This is particularly advantageous in complex defect morphologies such as the inverse ' $V$ '- shape seen in the present case.

Prosthetic rehabilitation of edentulous areas that are both horizontally and vertically deficient is one of the most complex treatment modalities in dentistry. Bone augmentation to reestablish the lost dimensions followed by implant supported prostheses is probably the only way that one that can effectively restore the pink and white aesthetics to as natural a result, as possible. Among the various techniques that have been introduced and used over the years for ridge augmentation, guided bone regeneration has gained a lot of prominence for its ease and predictability.

Vertical ridge augmentations presented with more challenges as there is no scaffold to receive the graft for possible guided bone regeneration. Thus, one needs to use a graft that is rigid enough to be stable on its own or use rigid barrier membranes such as those made of titanium to retain the intended shape of the ridge. Other techniques such as distraction osteogenesis, while having shown success, are rather difficult to employ in cases with small edentulous spans. Block grafts employ a bone or bone substitute material block to increase the width and/or height of a deficient residual ridge. These were made popular by Misch and Khoury. While Misch used a corticocancellous block obtained from the ramus, Khoury split the bone block into two thinner bone plates. ${ }^{18,19}$ These could be used to create an envelope within the walls of which the particulate graft could be packed, thus recreating the natural anatomy of the maxillary and mandibular bone. The extent of donor site morbidity was greater in Misch's technique as it involved harvesting a solid bone block from the ramus, the size of which depended on the size of the defect.

The current case utilised a variation of the Khoury's technique in that the cortical plates were obtained from the symphysis and the particulate graft was a combination of autogenous cancellous bone and freeze-dried bone allograft FDBA. The symphysis was selected as the donor site due to its proximity to the recipient site. The symphysis could accommodate the intended dimensions of the cortical shells while maintaining a safe distance from the mental foramina and the apices of the mandibular anterior teeth. A piezo-surgical insert was used in the current case as it was judged to be more conservative and less traumatic for the patient.

Placement of the cortical shells and the means to secure these have also been subject to considerable variation. Stimmelmayer placed one of the shells horizontally to approximate the future position of the alveolar crest. ${ }^{16}$ Gluckman and Du Toit placed the cortical shells on either side of the defect and secured these with three titanium screws with the central screw going across the defect space. ${ }^{17}$ The positions of the cortical shells may be altered as desired to obtain the intended horizontal as well as vertical augmentations using the titanium screw that anchors these shells. In the current case, a single titanium screw was placed across the defect space to snugly secure the shells. This was done primarily to avoid impinging on the nasopalatine nerve and vessels that emerged at the mesial end of the defect.

The choice of particulate graft depends on the availability of bone at the donor site, the morbidity expected, the patient's general health, the size of the defect etc. While autogenous bone is the most desirable due to its osteoconductive, osteoinductive and osteogenic potential, harvesting the required quantity to graft a sizeable defect might lead to a significant amount of morbidity. Allografts may help overcome this but also result in longer healing times. Stimmelmayr et al., in a similar technique, achieved horizontal ridge augmentation using autogenous bone harvested from mandibular ramus. ${ }^{14}$ In the current case, a combination of autogenous cancellous bone scrapings along with FDBA was used to pack in the space between the cortical shells. 
The maxillary anterior region is a particularly sensitive area as it is not only the most susceptible to trauma and rapid bone resorption but also the most critical in terms of a patient's smile, facial appearance, phonetics and an incisal guidance. A low lip line might prove to be slightly forgiving when it comes to developing an ideal emergence profile, which is a challenge when coupled with the need for vertical and horizontal augmentations. In the current case, the incisive foramen was particularly large and opened at the crest at the mesial end of the defect. In such cases, placing an implant in an ideal prosthetic position might not be possible and one might need to augment the ridge even in the presence of adequate ridge width.

\section{CONCLUSION}

This case reports a modified technique for augmentation of $3 \mathrm{D}$ defects. It can be stated that the use of this technique is a predictable method for augmentation of defects with complex configuration. With the use of particulate graft material, this treatment modality potentially reduces time for augmentation and formation of vital alveolar bone. A mixture of autograft and allograft can be successfully used in cases with deficient autograft availability.

\section{CONFLICT OF INTEREST}

The authors declared that there is no conflict of interest.

\section{REFERENCES}

1. Pietrokovski J, Massler M. Alveolar ridge resorption following tooth extraction. J Prosthet Dent. 1967 Jan;17(1):21-7.

2. Chappuis V, Araújo MG, Buser D. Clinical relevance of dimensional bone and soft tissue alterations post-extraction in esthetic sites. Periodontol 2000.2017 Feb;73(1):73-83.

3. Grunder U, Gracis S, Capelli M. Influence of the 3-D bone-to-implant relationship on esthetics. Int J Periodontics Restorative Dent. 2005 Apr;25(2):113-9.

4. Moro A, Gasparini G, Foresta E, Saponaro G, Falchi M, Cardarelli L, De Angelis P, Forcione M, Garagiola U, D’Amato G, Pelo S. Alveolar Ridge Split Technique Using Piezosurgery with Specially Designed Tips. Biomed Res Int. 2017;2017:4530378.

5. Benic GI, Thoma DS, Jung RE, Sanz-Martin I, Unger S, Cantalapiedra A, Hämmerle CHF. Guided bone regeneration with particulate vs. block xenogenic bone substitutes: a pilot cone beam computed tomographic investigation. Clin Oral Implants Res. 2017 Nov;28(11):e262-e270.

6. Benic GI, Joo MJ, Yoon SR, Cha JK, Jung UW. Primary ridge augmentation with collagenated xenogenic block bone substitute in combination with collagen membrane and rhBMP-2: a pilot histological investigation. Clin Oral Implants Res. 2017 Dec;28(12):1543-1552.

7. Tanaka K, Sailer I, Kataoka Y, Nogami S, Takahashi T. Sandwich bone graft for vertical augmentation of the posterior maxillary region: a case report with 9-year followup. Int J Implant Dent. 2017 Dec;3(1):20. doi: 10.1186/s40729-017-0063-9. Epub 2017 May 19.

8. Nogueira RLM, Osterne RLV, Abreu RT, Araújo PM. Alternative Distraction Osteogenesis Technique After Implant Placement for Alveolar Ridge Augmentation of the Maxilla. J Oral Maxillofac Surg. $2017 \mathrm{Jul} ; 75(7): 1402$.e1-1402.e8.

9. Jegham H, Masmoudi R, Ouertani H, Blouza I, Turki S, Khattech MB. Ridge augmentation with titanium mesh: A case report. J Stomatol Oral Maxillofac Surg. 2017 Jun;118(3):181-186. doi: 10.1016/j.jormas.2017.03.001. Epub 2017 Mar 28.

10. Elnayef B, Monje A, Gargallo-Albiol J, Galindo-Moreno P, Wang HL, Hernández-Alfaro F. Vertical Ridge Augmentation in the Atrophic Mandible: A Systematic Review and Meta-Analysis. Int J Oral Maxillofac Implants. 2017 Mar/Apr;32(2):291-312.

11. Deeb GR, Tran D, Carrico CK, BlockE, Laskin DM, Deeb JG. How Effective Is the Tent Screw Pole Technique Compared to Other Forms of Horizontal Ridge Augmentation? J Oral Maxillofac Surg. 2017 Oct; 75(10):2093-2098.

12. Li X, Xu P, Xu X, Liu S. The application of a delayed expansion technique for horizontal alveolar ridge augmentation in dental implantation. Int J Oral Maxillofac Surg. 2017 Nov;46(11):1451-1457.

13. Mounir M, Mounir S, Abou-Elfetouh A, Shaker MA. Assessment of vertical ridge augmentation in anterior aesthetic zone using onlay xenografts with titanium mesh versus the inlay bone grafting technique: A randomized clinical trial. Int J Oral Maxillofac Surg. 2017 Nov;46(11):1458-1465.

14. Stimmelmayr M, Güth JF, Schlee M, Göhring TN, Beuer F. Use of a modified shell technique for three-dimensional bone grafting: description of a technique. Aust Dent J. 2012 Mar;57(1):93-7.

15. 15. Khoury F, Khoury CH. Mandibular bone block grafts: instrumentation, harvesting technique and application. J Par ImplOrale. 2006;25:15-34.

16. Stimmelmayr M, Güth JF, Schlee M, Beuer F. Vertical ridge augmentation using the modified shell technique--a case report. J Oral Maxillofac Surg. 2014 Feb;72(2):286-91.

17. Gluckman H, Du Toit J. Reconstruction of a single-tooth traumatic defect in the anterior maxilla using the Khoury bone plate graft. Int Dent Afr Ed 2015;5:60-70.

18. Misch CE, Dietsh F. Autogenous bone grafts for endosteal implants--indications and failures. Int J Oral Implantol. 1991;8(1):13-20.

19. Khoury F, Antoun H, Missika P. Bone augmentation in oral implantology. UK: Quintessence; 2007. 